Pourquoi chanter les ragots du passé ? Itinéraire historique d'un chant rituel trumai (Mato Grosso, Brésil)

\title{
Emmanuel de Vienne
}

\author{
(2) OpenEdition \\ Journals \\ Édition électronique \\ URL : http://journals.openedition.org/jsa/11762 \\ DOI : 10.4000/jsa. 11762 \\ ISSN : 1957-7842 \\ Éditeur \\ Société des américanistes \\ Édition imprimée \\ Date de publication : 5 octobre 2011 \\ Pagination : 291-319 \\ ISSN : 0037-9174 \\ Référence électronique \\ Emmanuel de Vienne, «Pourquoi chanter les ragots du passé ? Itinéraire historique d'un chant rituel \\ trumai (Mato Grosso, Brésil) », Journal de la société des américanistes [En ligne], 97-1 | 2011, mis en \\ ligne le 10 décembre 2014, consulté le 11 mars 2020. URL : http://journals.openedition.org/jsa/11762 \\ ; DOI : https://doi.org/10.4000/jsa. 11762
}




\title{
POURQUOI CHANTER LES RAGOTS DU PASSÉ ? ITINÉRAIRE HISTORIQUE D'UN CHANT RITUEL TRUMAI (MATO GROSSO, BRÉSIL)
}

\author{
Emmanuel DE VIENNE*
}

Le rituel du manioc, spécificité trumai dans le système plurilingue et pluriethnique du Haut Xingu, est déclaré perdu depuis des décennies. Il constitue, pour cette raison, un élément important de la rhétorique nostalgique par laquelle se construit l'identité du groupe. Certains de ses chants ont cependant été énoncés en 2006 à l'occasion de l'inauguration d'une infirmerie dans un poste FUNAI. En comparant cet événement avec le rituel tel qu'il est décrit dans la littérature et par les Trumai, il apparaît que ce sont curieusement les chants les moins valorisés qui ont été ainsi promus au rang d'emblème culturel : des réponses chantées aux ragots et aux accusations. Le présent article identifie les raisons de ce choix et de ce changement de statut, et cherche ainsi à éclairer des processus de constitution et de transformation des traditions que la catégorie de patrimonialisation tend à uniformiser. [Mots-clés: Amazonie, Haut Xingu, tradition, rituel, patrimonialisation, cultura, relations interethniques.]

Porque cantar as fofocas do passado ? Itinerário histórico de um canto ritual trumai. O ritual da mandioca, uma especialidade trumai no contexto do sistema plurlinguístico e pluriétnico do Alto Xingu, é considerado perdido há décadas. Por esta razão, o ritual constitui um elemento importante na retórica nostálgica a partir da qual se constrói a identidade do grupo. Alguns dos cantos do ritual foram, no entanto, entoados em 2006, durante a inauguração de uma posto de saúde no posto da FUNAI. Comparando este evento com o ritual tal como é descrito na literatura e pelos Trumai, parece, curiosamente, que são justamente os cantos menos valorizados que foram promovidos ao estatuto de emblema cultural : as respostas cantadas às fofocas e às acusações. Este artigo identifica as razões desta escolha e desta mudança de estatuto, e busca assim esclarecer os processos de constituição e de transformação das tradições que tendem a ser negligenciadas através da categoria de "patrimonialização ». [Palavras chaves : Amazônia, Alto Xingu, tradição, ritual, patrimonialização, cultura, relações interétnicas.]

Why to sing the gossips of the past? Historical itinerary of a Trumai ritual song. The Trumai manioc ritual has been declared lost for decades. As such, it is a

* Laboratoire d'anthropologie sociale, 52 rue du Cardinal Lemoine, 75005 Paris [emmanueldevienne@googlemail.com].

Journal de la Société des Américanistes, 2011, 97-1, pp. 291-319. C Société des Américanistes. 
key element in the nostalgic rhetoric upon which group identity is built in contrast with the other groups forming the Upper Xingu system. Nevertheless in 2006 some of its songs were performed in the opening ceremony of a infirmary in a FUNAI post. Compared with the way the ritual is described both in literature and by the Trumai, this event shows that, surprisingly enough, the songs held up as cultural emblems were the least valued ones, consisting in sung answers to gossip and accusations. The present article identifies the reasons behind this choice and this changing status, thus highlighting processes at work in the constitution and transformation of traditions that the category of patrimonialization tends to make uniform. [Key words : Amazonia, Upper Xingu, tradition, ritual, patrimonialization, cultura, interethnic relations.]

Analyser les chants rituels chez les Trumai (Mato Grosso, Brésil) pose un problème élémentaire : leur mode d'existence est presque exclusivement celui du souvenir nostalgique. Les Trumai sont connus, dans la société plurilingue du Haut Xingu, pour ne plus pratiquer aucun rituel depuis plus de vingt ans, faute, selon leur propre point de vue, de structuration sociale «normale » à même d'assurer l'organisation des rituels et la transmission du savoir spécialisé et valorisé que cela implique. La plupart des chants rituels sont réputés irrémédiablement "perdus » et " oubliés » depuis la mort de l'ancien chef et grand spécialiste rituel, Amilcar ${ }^{1}$ dans les années 1990. Dans ce contexte, l'énonciation en 2006 des chants du manioc (ole wal) par une femme trumai lors de l'inauguration d'une infirmerie dans un poste FUNAI $^{2}$ ne peut qu'attirer l'attention. Cet article a pour objet d'analyser cet événement dans toute sa complexité. Comment l'aborder alors même que son «contexte total»-pourtant jugé essentiel depuis les travaux fondateurs de Seeger $(1979,1987)$ - est, de l'aveu même des Trumai, radicalement nouveau et «non traditionnel»? Cette différence invite en premier lieu à n'envisager l'événement que comme le résultat d'un mouvement désormais mondial d'objectivation et de patrimonialisation des cultures autochtones. De fait, lors des inaugurations de structures collectives dans le Parc national du Xingu, il est maintenant d'usage que chacun offre, dans un rituel syncrétique, un échantillon de sa " cultura » aux autres. Ces facteurs massifs, pour importants qu'ils soient, sous-déterminent pourtant la forme que prend cette « revitalisation ». Pourquoi est-ce la fête du manioc, qui a cessé d'être pratiquée bien avant les autres (elle était déjà dans les années 1960 un objet du passé), qui fait l'objet d'une telle tentative ? Pourquoi surtout, au sein des chants du manioc, est-ce un sous-genre vocal qui a été choisi, celui des chants diurnes ? Le rituel du manioc impliquait en effet deux types de chants qui différaient quant à ce que Déléage (2005) appelle leur « épistémologie », c'est-à-dire l'ensemble des représentations qui les accompagnent et spécifient leurs conditions d'apprentissage et d'énonciation. Alors que les chants diurnes étaient en trumai, ouverts à l'innovation, et traitaient des rumeurs et des conflits locaux, les chants nocturnes - de loin les plus valorisés - étaient incompréhensibles et formaient un répertoire 
idéalement clos dont l'apprentissage était réglé. Or ce sont les premiers - les ragots du passé - qui sont curieusement choisis aujourd'hui par un village trumai pour être exhibés comme cultura auprès de leurs voisins.

Cet article se propose d'identifier les raisons de ces choix successifs, en montrant qu'ils ne peuvent être réduits à un processus univoque de patrimonialisation ou d' "invention de la tradition » dans un contexte mondialisé. $\mathrm{Au}$ contraire, ils permettent d'éclairer un certain nombre de questions importantes : comment s'articulent au cours du temps le chant rituel (un contenu) et son institution, c'est-à-dire le contexte rituel dans lequel il est énoncé ? Quels sont les facteurs sociopolitiques qui contraignent l'évolution de ce rapport et conditionnent de ce fait la mémoire, l'oubli ou l'invention du savoir rituel ? On commencera par donner des éléments de contexte sur les Trumai et la fête du manioc avant de comparer l'occurrence récente avec le contexte d'énonciation « traditionnel », en se fondant en partie sur les descriptions fournies par l'anthropologue américain Buell Quain en 1938 et publiées par Murphy en 1955 (Murphy et Quain 1955).

\section{Les Trumai, la cultura et la fête du Manioc}

La «culture » (cultura), notion d'importation désormais omniprésente en Amazonie, a chez les Trumai une effectivité singulière. C'est en effet sa « perte » qui sature la parole des intéressés, à savoir l'impossibilité à donner à voir sa manifestation prototypique que sont le rituel et les différents savoirs objectivés qu'il mobilise : plumasserie, chorégraphies, mais surtout musiques vocales et instrumentales. Cette perte détermine, pour une large part, les rapports qu'entretiennent les Trumai avec leurs voisins, notamment ceux qui participent au système pluri-ethnique du Haut Xingu. Ce système rassemble dix groupes de langues différentes et, souvent, mutuellement inintelligibles : les Kuikuro, les Kalapalo, les Matipu et les Nafukwa (de langue caribe), les Yawalapiti, les Mehinaku et les Wauja (de langue arawak), les Aweti et les Kamayura (de langue tupi) et, partiellement, les Trumai, dont la langue est un isolat linguistique (Monod Becquelin 1975). Cet ensemble est caractérisé, en dépit des différences linguistiques, par une homogénéité culturelle remarquée dès la première description de la région par Steinen en 1884 (Steinen 1886). Les frontières externes de cet ensemble sont dynamiques et peuvent progressivement intégrer des voisins auparavant vus comme "féroces » et donc contraires à l'idéal pacifique xinguanien. Les Kisêdje (anciennement Suyá) et les Ikpeng (anciennement Txicão) sont, à cet égard, les derniers à avoir en partie été ainsi pacifiés. Arrivés tardivement dans la région, dans la première moitié $\mathrm{du} \mathrm{XIX}^{\mathrm{e}}$ siècle, les Trumai sont toujours restés dans une position marginale, qui se traduit sur la longue durée par une oscillation à la fois résidentielle et matrimoniale entre le Haut Xingu (en particulier les Kamayura) et le Bas Xingu (en particulier les Suyá). La peur des sorciers du Haut Xingu et des guerriers du Bas Xingu constitue la motivation principale de ce qu'il 
faut bien qualifier de fuites successives sur près de 200 ans. On compte ainsi pas moins de 19 villages différents qui furent fondés durant cette période, un chiffre encore inférieur à celui des déménagements puisqu'un même village peut avoir été habité à des périodes différentes. Trois facteurs (les guerres, les épidémies et le pillage des femmes par le raid ou l'intimidation) expliquent les goulots d'étranglement démographique à l'époque de Quain (Murphy et Quain 1955, p. 20) en 1938 et de Monod Becquelin (1975) en 1967, où la population n'excédait pas la trentaine d'individus. Ces facteurs sont eux-mêmes corrélés au taux de mariages interethniques, beaucoup plus élevé que dans les autres groupes (à l'exception notable des Yawalapiti). À partir des années 1980, si la croissance démographique commencée une dizaine d'années plus tôt s'est confirmée ${ }^{3}$, elle est allée de pair avec de nombreuses scissions. Celles-ci se sont accélérées après la mort du chef Amilcar dans les années 1990, au point qu'aujourd'hui les unités locales regroupent entre 15 et 35 individus seulement, bien loin des 300 à 400 que peuvent rassembler les grands villages circulaires xinguaniens. Une telle différence d'échelle implique des différences qualitatives : aucun village trumai ne reproduit complètement la structure spatiale idéale, à commencer par la maison des hommes où sont normalement conservées les flûtes masculines $k u \tau^{4}$. Pour cette raison en particulier, aucun Trumai ne qualifie de «village » véritable (ilaka) les unités locales. Et cette absence compte beaucoup, selon eux, dans la disparition des pratiques rituelles collectives, presque à égalité avec le défaut de savoir spécialisé.

Cette situation atypique se traduit par trois antiennes assumées par leurs voisins aussi bien que par les Trumai eux-mêmes : ils « bougent sans arrêt », " n'arrêtent pas de se disputer entre eux » et « n'ont plus de cultura ». Les trois sont liées, qui reflètent respectivement le nomadisme lié aux guerres et à la sorcellerie, l'éclatement géopolitique et la cessation des activités rituelles. Les trois tranchent de façon massive avec, respectivement, la sédentarité xinguanienne, l'ethos d'entraide et de pacifisme entre co-résidents et l'opérateur indispensable de l'articulation entre les unités du système inter-ethnique. Ces étiquettes essentialistes n'en continuent pas moins d'être projetées sur les Trumai de la même manière que d'autres le sont sur les autres groupes (« les X sont menteurs », « les Y cuisent à peine leur poisson », etc.), signe que cette personne morale continue d'être pertinente. On le doit sans doute au maintien, toutefois fragile, d'une langue propre, qui demeure le critère diacritique principal des unités du système.

L'interethnicité produit de la réflexivité, qui est elle-même mère du paradoxe, comme le remarque Carneiro da Cunha en citant Bertrand Russell (in Carneiro da Cunha 2010, p. 79). La définition des Trumai est ainsi essentiellement paradoxale. Faisant indéniablement partie du Haut Xingu (généalogiquement, matrimonialement, politiquement), ils sont tributaires d'une série de normes qu'ils violent malgré eux systématiquement sans pour autant être dissous en tant qu'unité sociale : ils sont ainsi l'ethnie qui n'en est pas une (« les Trumai n'existent plus ») et dont la «culture » est de n'en avoir plus. L'adhésion au groupe est 
formulée par une rhétorique émotionnelle de la nostalgie et de l'empathie non moins paradoxale, mais dont l'efficacité est indéniable. On est Trumai par la nostalgie commune à ne plus pouvoir l'être pleinement en exhibant une cultura vivace et dans la mesure où ce manque s'enracine dans une incapacité à vivre ensemble, on se rassemble dans les discours autour de l'expérience partagée d'une impossibilité à se rassembler.

La fête du manioc (ole deani) est une pièce essentielle de cette rhétorique nostalgique, en tant qu'il s'agit du rituel à la fois le plus «perdu » et le plus « trumai ». De très nombreuses conversations tournent autour de projets de documentation du savoir rituel auprès des plus âgés et de mise en pratique par la construction d'un village destiné à servir de scène rituelle. Ma propre enquête de terrain est partie prenante de cette dynamique patrimoniale, puisqu'elle a été financée par le Max Planck Institute de Nimègue dans le cadre d'un projet DoBeS (Documentation des langues en danger) coordonné par Monod Becquelin et Guirardello-Damian ${ }^{5}$. De tous les rituels que l'on ne fait plus (c'est-à-dire tous à l'exception de timides performances en 2007 et 2008 des clarinettes takwara, rituel le plus quotidien et dont la maîtrise est la mieux partagée), la fête du manioc est la plus regrettée. Selon les Trumai d'aujourd'hui, il n'est plus possible d'espérer la faire à nouveau, car la transmission des chants s'est interrompue, le dernier chanteur et ancien chef étant mort sans l'avoir enseigné à quiconque. Dès mon arrivée, le fait qu'Aurore Monod Becquelin en avait enregistré les chants lors de son terrain à la fin des années 1960 a été évoqué avec excitation, puis à de nombreuses reprises tout au long de mes séjours. Le retour de ces enregistrements devait permettre d'apprendre le rituel pour le faire à nouveau. Ce scénario est néanmoins largement idéalisé. Ces enregistrements ont été en effet envoyés à plusieurs reprises au cours des dernières décennies sans avoir débouché sur une revitalisation. Une des raisons en est qu'Amilcar, selon les informations d'Aurore Monod Becquelin (communication personnelle), ne se présentait pas comme spécialiste de la fête du manioc. Il était en mesure d'en restituer certains chants, mais considérait déjà le rituel comme « perdu » à l'époque. Cette contradiction signale que le rituel ne fait pas l'objet d'un oubli progressif, mais tend à être toujours situé à la même distance, à la fois suffisamment proche pour être regretté et trop loin pour être accessible. Cette importance considérable au regard d'une absence de fait s'explique sans peine par le jeu des relations interethniques au sein de l'ensemble du Haut Xingu. Les divers groupes qui le composent se livrent à une compétition de prestige ayant principalement le rituel pour cadre et pour critère. Les grands rituels intertribaux sont l'occasion d'échanges somptuaires entre les villages qui présupposent qu'en leur sein soient établies des positions hiérarchiques différenciées entre ceux qui peuvent assumer la charge de la fête et ceux qui ne le peuvent pas. La patrimonialisation induite par le contact avec les Blancs intensifie encore cette focalisation rituelle. Une fête "traditionnelle » permet d'attirer journalistes, chercheurs, documentaristes, à la fois pour « pacifier » ces 
nouveaux étrangers, selon une logique préexistante (Fausto 2004 ; Fiorini et Ball 2006), et augmenter le flux de richesses qui nourrit le rituel.

À la différence du javari (hopep en trumai, javari étant le nom tupi transxinguanien) apporté par les Trumai et adopté par tous les groupes, la fête du manioc est restée l'apanage des Trumai et, donc, d'autant plus importante. Ils la définissent comme le « kwarup des Trumai », le kwarup étant un rituel de double funéraille (Agostinho da Silva 1974) pratiqué par tous les groupes du Haut Xingu à l'exception des Trumai. Ce temps fort de la vie intertribale, qui réunit plusieurs milliers de personnes, a en effet des ressemblances formelles immédiates avec l'ole deani. Tous deux s'articulent autour de troncs peints érigés sur la place, la différence, outre les motifs graphiques, étant la présence d'un tronc plus grand et tordu (nacha nacha) dans le rituel du manioc. La comparaison peut se faire aussi au niveau de la valeur emblématique de ces deux rituels. Le kwarup a acquis une célébrité à l'échelle du Brésil entier, devenant une sorte de rituel indigène générique dans l'imagerie nationale ${ }^{6}$, quand la fête du manioc est un emblème de « trumaïté ». Cette particularité des Trumai d'avoir un rituel en propre plutôt que de s'agréger au rituel commun à tous est révélatrice des rapports ambigus qu'ils entretiennent avec le Haut Xingu depuis leur arrivée dans la région. L'ancien chef, mort dans les années 1990, raconta à Aurore Monod Becquelin (communication personnelle) que la seule tentative que firent les Trumai pour adopter le kwarup provoqua le réveil des troncs, qui les chassèrent en les battant. Cette expulsion originelle du rituel haut-xinguanien principal les force à élever celui du manioc à la même position emblématique dans une compétition symétrique, alors que les autres groupes ont été invités à y participer. L'important pour eux aujourd'hui n'est pas de participer au kwarup, mais de pouvoir exhiber, dans la compétition de prestige que se livrent les groupes, une fête à la fois authentique et exclusive. Ce serait la plus belle revanche qu'ils pourraient prendre sur leurs voisins et parents qui disent souvent d'eux qu'ils «n'ont plus de culture » et "sont déjà devenus des Blancs ", au point même, selon certains, de ne plus mériter l'aide de la FUNAI.

Si ses chants sont perdus, le rituel fait donc mémoire. Mais une mémoire qui prend désormais la forme d'une image statique, celle des troncs peints alignés au pied desquels on dépose la nourriture rituelle. Une image objectivable par la peinture, puisqu'elle est un des thèmes de prédilection des peintres trumai. Amatiwana fut le premier de ces peintres dans les années 1970. Son exemple fut suivi - pour ce qui est des Trumai - par un de ses fils, par un petit-fils et par un neveu classificatoire dans un autre village. Dans un style figuratif naîf à la symétrie rigoureuse, tous se sont essayés au sujet, qui indexe moins un contexte rituel spécifique - et su par tous - qu'une trumaïté dont la pureté est garantie par l'ancienneté. Nul n'est surpris par le fait qu'une fête du manioc est un curieux emblème d'autochtonie pour un groupe qui se souvient n'avoir découvert ce cultigène qu'au contact du Haut Xingu (Villas Bóas et Villas Bóas 1974, p. 24). 


\section{UNE BRÈVE HISTOIRE DU RITUEL}

Ce en quoi consistait la fête du manioc est, dans ce contexte, une question épineuse. La seule description qu'on en ait dans la littérature est due à Murphy et Quain (1955, pp. 67-70), d'après les notes de terrain que ce dernier rédigea lors de son séjour chez les Trumai en 1938. Et ce qu'il en dit contredit en partie les explications actuelles. L'exercice auquel on se livre ici consiste donc à formuler - à partir d'une observation du rituel en 1938 - quelques hypothèses sur la façon dont ont évolué depuis les souvenirs de la fête du manioc. Dans l'ouvrage de Murphy et Quain (1955), cette « cérémonie » apparaît comme la plus importante et la plus fréquemment tenue chez les Trumai. Elle avait lieu presque quotidiennement de la mi-août à la fin du mois de septembre, période correspondant à la fin de la saison sèche, donc à la période du brûlis et des plantations. Sa fonction, selon Murphy et Quain (ibid.,p. 67), était essentiellement propitiatoire : « son but était principalement d'assurer une récolte de manioc abondante, et son symbolisme tournait clairement pour l'essentiel autour du thème de la fertilité ${ }^{7}$.

Selon les Trumai d'aujourd'hui, le rituel était moins long (quelques jours) et associé à la maladie. Il était organisé par les femmes qui étaient tombées malades à cause du maître du manioc, ole yar, appelé aussi nukekerihe. Cette maladie provoquée par le manioc était la condition pour devenir soi-même " maître » (yar) de la fête, c'est-à-dire assumer à la fois l'organisation et la charge économique de l'événement par des paiements en nourriture versés aux participants. Le manioc devait être identifié, comme aujourd'hui, par le rêve du malade et par la cure chamanique qui procède par extraction. Dans ces cas, le praticien extrait, puis montre au malade un morceau de branche de manioc ou un ver (mïrïr ïr), véritable ou modelé dans un matériau noirâtre. Ce ver est le parasite du manioc et un des avatars de l'esprit maître de la plante, tout comme une vieille femme (ayets ou atseda) en est la " grand-mère ». Si le terme denetsak désigne les " esprits » en général, y compris le maître du manioc, il cède sa place au terme honTal dès lors qu'est actualisée une relation singulière avec un humain par la maladie. C'est donc ainsi que l'on se réfère au « pathogène familier » d'un individu en particulier, tout comme à ses figurations rituelles (danseurs et chanteurs, troncs, masques, parures corporelles). Dans la «fête » (deani) du manioc, cet esprit maître était figuré par une série de troncs érigés sur la place du village, écorcés et peints sur une partie de leur hauteur (Figures 1 et 2), au pied desquels était déposée quotidiennement de la nourriture (galette de manioc et poisson). Les Trumai actuels donnent sur ce dispositif et sur son utilisation au cours du rituel des informations complémentaires de celles de Quain. D'abord, comme le remarquent ces derniers, l'un des troncs, nukekerehe ${ }^{8}$, faisait l'objet de plus d'attentions. Ilétait considéré comme « malin » et était formellement différent des autres, puisqu'il était « tordu » (nacha nacha) en zigzags, alors que les autres (désignés comme ole pi’tsi, « manioc véritable ») étaient droits et d'une section plus importante (Figure 3). Ce tronc représentait la 

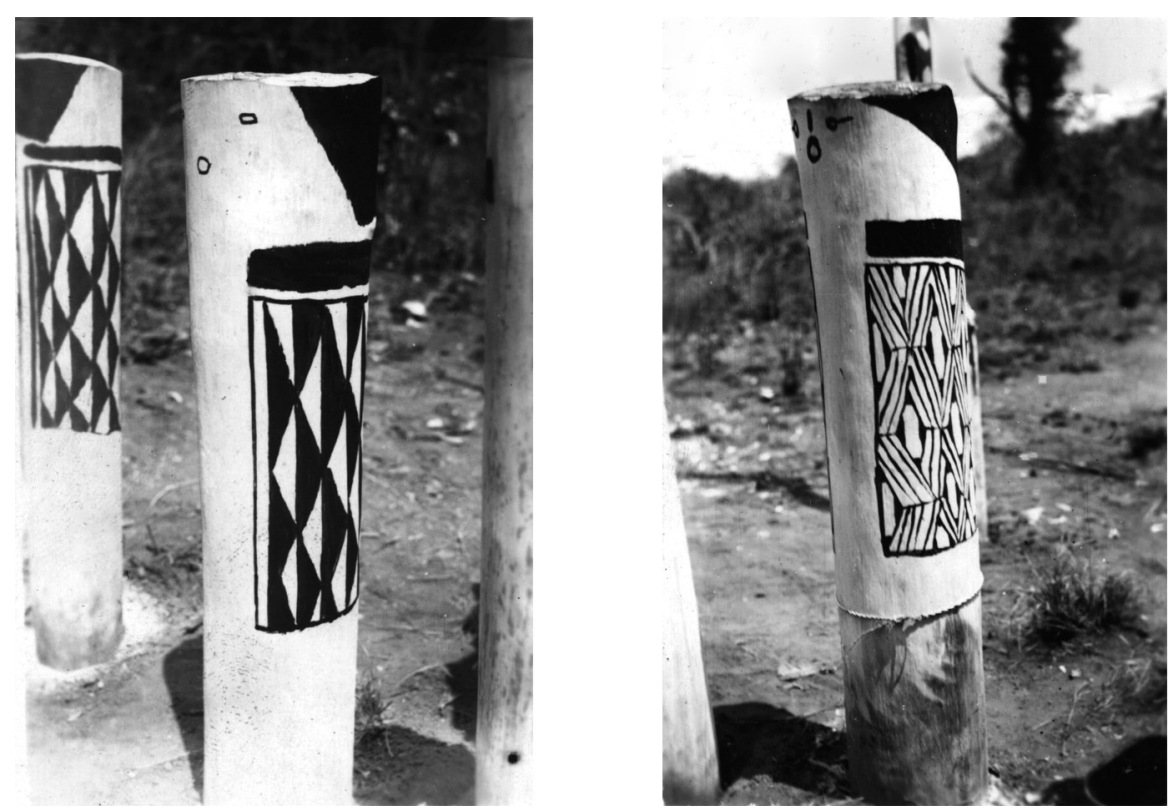

FIG. 1 et 2 - Poteaux figurant le maître du manioc ole yar (photo Buell Quain 1938) ${ }^{9}$.

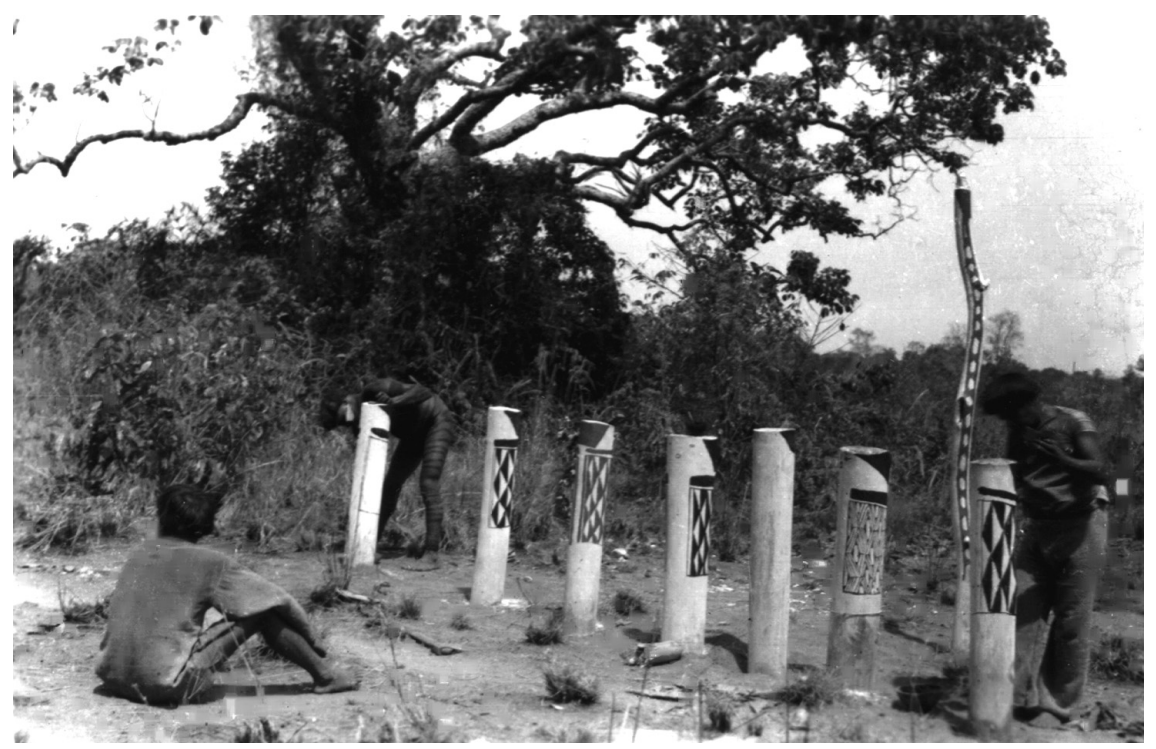

FIG. 3 - Les effigies sont disposées dans une clairière pour recevoir de la part des seuls hommes une décoration préliminaire. Leur transport ensuite jusqu'au village s'accompagne d'un chant et d'une danse (photo et légende Buell Quain 1938 : The effigies are arranged in a forest clearing for preliminary decoration - by the men only. Later they will be carried into the village with a song and a dance). 
vieille femme atseda, qui était wech (jalouse/avare) et à qui il fallait donner «beaucoup d'atsaek et de maisawari [deux types de soupe chaude et sucrée de manioc (portugais, noté Pt., perereba)] et des chants pendant cinq ou six nuits, sans quoi elle emmenait les humains dans la forêt ».

Murphy et Quain identifièrent deux types de chants. Les premiers étaient collectifs : un chanteur principal, parfois accompagné d'un autre homme, guidait de maison en maison une procession de danseuses qui fournissaient également les répons. Les seconds étaient individuels, sans chœur, et pouvaient " même » être chantés par les femmes. Là aussi, le chanteur se rendait dans toutes les maisons à tour de rôle, pour chanter et danser. Cette opposition est confirmée par les Trumai. Ils la formulent en qualifiant les premiers de chants nocturnes, les seconds de chants diurnes (tamulan chïk). Il est possible que cette séparation n'ait pas été si tranchée et que l'ensemble des chants ait été pris dans une séquencialité moins binaire, calquée sur les différentes phases de la journée, à la manière dont procède par exemple le javari (Menezes Bastos 2003). Quand bien même cette dichotomie par le moment de l'énonciation serait rétrospective et donc sujette à caution, reste que la comparaison entre les données de Quain et les discours contemporains confirme l'existence de deux genres vocaux différents, auxquels on se référera par commodité en reprenant cette opposition entre diurnes et nocturnes. Chaque tronc avait un maître (yar) humain, exclusivement féminin d'après les informations actuelles, qui était responsable des offrandes déposées devant lui. La maîtresse principale de la fête était responsable de nukekerihe, les autres se répartissant les « vrais manioc » selon un ordre dont la logique est inconnue. Il est difficile d'établir également si toutes ces femmes assumaient ce rôle rituel en vertu d'une maladie antérieure ou si cette forme d'élection était réservée au maître principal. Un paragraphe de Murphy et Quain (1955, p. 70) fait référence à une relation rituelle désignée par la locution ole aton, aton signifiant « animal familier ».

[...] mais dans ce contexte il désigne une identification cérémonielle temporaire, d'une part entre des personnes, de l'autre entre des personnes et les poteaux de manioc. Le lien interpersonnel ainsi établi ne pouvait unir que des individus de sexe opposé. Cependant un homme pouvait avoir pour aton plusieurs femmes, et réciproquement. ${ }^{10}$

Les informateurs actuels mentionnent que chaque tronc était aton de la personne qui avait la charge des offrandes déposées à son pied. Cette personne était logiquement « maître » (yar) de ce tronc. La relation yarlaton, qui concerne normalement un humain et un animal (typiquement un oiseau), s'accompagne d'une obligation de soin et d'attention. Le mauvais traitement et la négligence envers un animal familier sont réprouvés et réputés provoquer une vengeance post mortem puisque, dans le village des morts, ce sont les aton qui prennent soin de leurs anciens maîtres. Le rituel du manioc donnait ainsi à voir une logique de 
familiarisation transitive : on s'assurait le contrôle du manioc en devenant le maître de son maître ${ }^{11}$.

La relation yar/aton entre humains à laquelle Murphy et Quain font référence ne trouve pas d'écho dans les souvenirs des Trumai d'aujourd'hui. Il est cependant probable et cohérent que la personne considérée comme yar d'un tronc fut aussi considérée comme yar des danseurs qui venaient ensuite consommer les offrandes, surtout que ces danseurs étaient eux aussi, comme les troncs, des figurations du manioc. S'il est impossible de déterminer avec certitude quelles relations de parenté sous-tendaient le choix des danseurs, il est permis de supposer, par homologie avec d'autres rituels xinguaniens ${ }^{12}$, qu'ils se recrutaient dans une autre maisonnée que celle du maître-mécène.

Cette relation n'était pas la seule à être mobilisée, comme le montre l'importance du genre. Le tronc lui-même était une image d'hermaphrodisme par les parures « corporelles » qui lui étaient appliquées. Chaque tronc était peint par un homme à l'extérieur du village ${ }^{13}$ (Figure 3). Même si Murphy et Quain ne le précisent pas, il est plausible que ce fut par l'époux de la femme qui était yar du tronc, donc yar lui-même. Cet acte de peinture rappelle les peintures corporelles que les hommes se peignent mutuellement, en l'absence des femmes, avant les rituels. Chaque tronc est ensuite porté sur la place du village par l'homme qui l'a peint. Là il reçoit de la part des femmes une peinture au roucou sur la base et au sommet, de la même manière que les femmes se peignent dans le Haut Xingu les pieds et le front (Figure 4). Cette peinture est donc féminine. Puis « hommes et femmes déposent du coton non filé sur leur sommet » (Murphy et Quain 1955, p. 69).

Une fois terminé et dressé sur la place du village, le tronc donnait ainsi à voir une contradiction : à la fois masculin et féminin, inerte et animé, objectivant le couple qui en était "maître », tout en en étant ontologiquement distinct. On retrouve ultérieurement cette ambivalence sexuelle puisque l'esprit du manioc, appelé habituellement « veille femme », se voyait incarné par un vieillard :

Un jour, Matiwana réalisa un rituel où il jouait apparemment le rôle de Nukekerihe. En poussant des cris de colère il sauta devant le tronc, au pied duquel se trouvaient deux bols de soupe de manioc. Il entonna alors un chant au sujet du tronc, que Nituary et les femmes reprirent avec lui. Après deux répétitions les femmes lui versèrent le contenu du bol sur la tête et les épaules, et il se trouva dégoulinant de soupe. Il se retira dans l'hilarité générale. Le second bol fut versé ensuite sur le poteau. Je ne pus obtenir d'explication complète sur les croyances qui entourent les poteaux du manioc. (ibid., p. 70) ${ }^{14}$

Un vieillard sauvage et agressif se voyait donc congédié et ridiculisé par l'aspersion d'une « soupe de manioc » ${ }^{15}$ par les femmes, et provoquant l'hilarité de l'assistance. «La peur provoquée par Nukekerihe était mêlée de plaisanteries » ${ }^{16}$, nous disent Murphy et Quain (ibid., p. 69), en donnant d'autres exemples : « une nuit, ils dirent à Quain que les hommes n'iraient pas se coucher avant lui, car s'il veillait tard tout seul Nukekerihe le mangerait certainement ${ }^{17}$ (ibid.). 


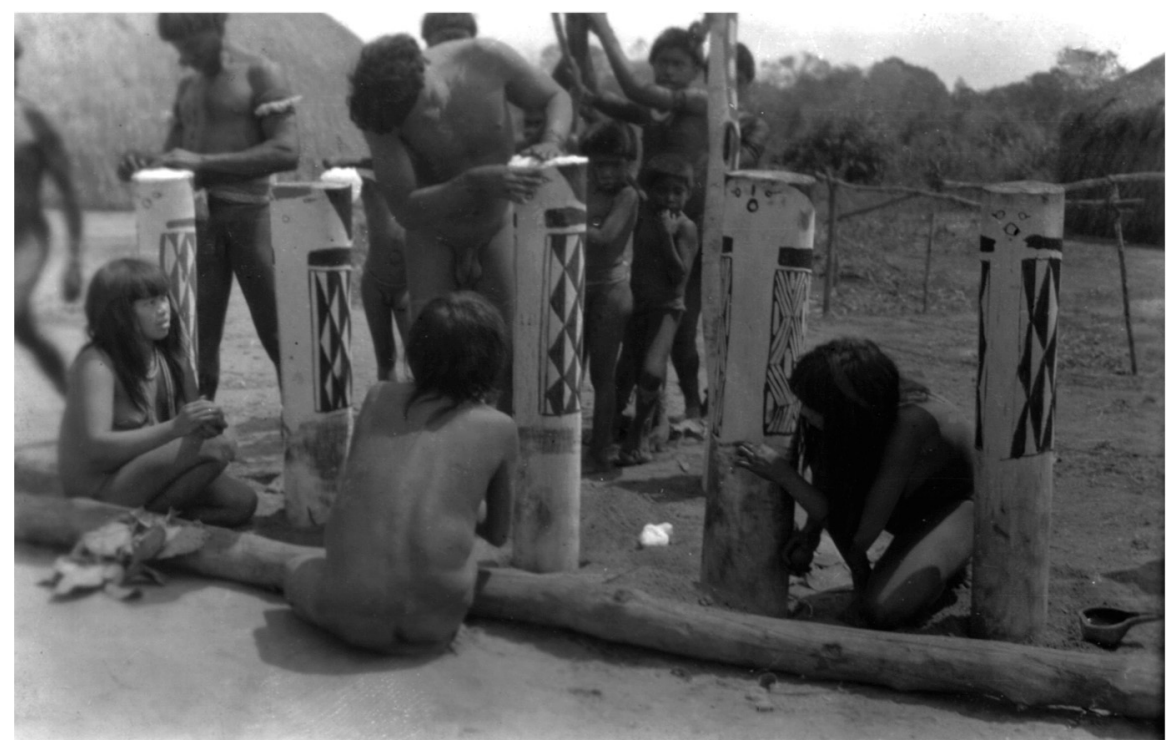

FIG. 4 - Les photos de la danse au cours de laquelle l'effigie est amenée au village ont été perdues dans la rivière. Une fois installée au centre du village, chaque effigie reçoit une perruque de coton. Les femmes appliquent avec du roucou les touches décoratives finales (photo et légende Buell Quain 1938 : Pictures of the dance which brought the effigies into the village were lost in the river. After the effigies are established in the center of the village, each is supplied with a wig of cotton. The women add final decorative touches with urucum).

Ces plaisanteries, en particulier la saynète d'aspersion, jouaient sans doute un rôle important dans l'attitude démontrée envers les troncs. Le statut d'agent accordé aux objets, qu'il est facile d'imputer comme une croyance, est en fait foncièrement ambigu. En réponse à la question : « donnait-on de la nourriture aux troncs? », on m'adressa un très prosaïque «comment est-ce que le tronc pourrait manger quoi que ce soit? ». Il était l'objet d'un pretend play dont la limite de pertinence était donnée par le cadre rituel, et personne, par définition, n'en était dupe. Le rire venait en premier lieu pointer et questionner cette zone d'indécision instaurée par les actes rituels. Mais il était aussi l'instrument et le signe d'un rapprochement de l'étranger. La relation à plaisanterie paradigmatique est celle qui s'instaure entre amipine, cousins croisés, une relation qui est appliquée par défaut aux visiteurs brésiliens ou étrangers. On la retrouve sans surprise dans la familiarisation dont faisaient l'objet les diverses figurations du manioc.

Comment interpréter l'ambivalence sexuelle? Elle reflète sans doute la division sexuelle du travail qui préside à la culture du manioc, division que la séquence des actions rituelles rejoue, chaque sexe en jouant sa part et invitant l'autre à l'action complémentaire, dans une «domestication» mutuelle. 
L'essartage, tâche masculine, s'oppose à la récolte et à la préparation culinaire, tâches féminines, une séparation qui fait du manioc, sous ses formes successives (de l'essart à la galette cuite), l'objet d'un échange entre époux. Dans le rituel, les hommes préparaient les troncs et consommaient seuls, devant les troncs, la nourriture préparée et déposée par les femmes (ibid., p. 69). Cette offrande ne peut donc pas être interprétée seulement comme un effort de familiarisation $\mathrm{du}$ manioc par la commensalité. La relation yarlaton se doublait d'une conjugalité selon laquelle le maître du manioc était par définition d'un autre sexe qu'ego. Si le tronc était masculin par les hommes et féminin par les femmes pendant sa fabrication, il était bien masculin pour les femmes et féminin pour les hommes dans la suite du rituel. Le point de vue féminin est relativement clair : c'était la femme yar du tronc qui déposait à son pied une nourriture consommée par des hommes identifiés par contiguïté spatiale au ole. Et c'étaient les femmes qui aspergeaient de manioc le vieillard Matiwana. Le point de vue masculin était-il rendu manifeste par le retour de la nourriture rituelle dans sa maison par le récipiendaire ? Cette question, plausible au regard d'autres rituels, où l'on ne consomme sur place qu'un échantillon du don pour en emporter l'essentiel chez soi avec ses co-résidents, n'est pas mentionnée par Murphy et Quain (1955). Elle indiquerait qu'au cours de sa circulation, la même nourriture était vue par celui qui la donnait comme adressée au ole, et par celui qui la recevait, lorsque lui-même la donnait à sa famille, comme provenant du ole : elle venait d'un tronc qui était la délégation du couple producteur. La trajectoire univoque et explicite de la familiarisation se compliquait ainsi d'une réversibilité et d'une complémentarité qui caractérisent la forme sociale que prennent localement la culture et la préparation du manioc.

La divergence entre les données de Murphy et Quain (1955) et celles que l'on obtient aujourd'hui (place de la maladie, relation yarlaton) ne vaut pas contradiction. Les catégories fonctionnelles classiques (thérapeutique, propitiatoire, prophylactique...) ne sont évidemment pas mutuellement exclusives. Il est probable que l'association avec la maladie a échappé à Quain en raison du fait que le malade maître de la fête était sans doute guéri depuis longtemps au moment où elle avait lieu. Si la spécificité de son rôle rituel était décelable, il était difficile de deviner, sans parler la langue et sans rester sur place longtemps, qu'il trouvait son origine dans un épisode pathologique. Inversement, il est possible que le rituel lui-même, tout comme son évocation aujourd'hui après plusieurs décennies d'éclipse, se soit infléchi vers le modèle des rituels thérapeutiques collectifs des Wauja qui sont voisins et parents des Trumai. Certains informateurs ne font plus guère de différence entre le rituel du manioc trumai ole wal et le honTal pitaka (littéralement "faire sortir l'esprit pathogène ». Ball 2011), dès lors qu'il concerne ce même esprit. Dans ce rituel, l'esprit pathogène (quel qu'il soit, y compris donc le manioc) est figuré par des chanteurs et des danseurs que l'on fait sortir de la maison des hommes, sur la place du village, et que l'on fait danser et 
chanter jusqu'à la maison du malade, où ils établissent avec lui un rapport de reconnaissance visuelle et dialogique mutuelle. Il peut prendre une forme rudimentaire et hâtive à l'apogée de la maladie, lorsque l'urgence de la situation exige une intervention immédiate, pour se répéter ultérieurement plus complètement (en nombre de participants, en durée et en importance économique et esthétique). Chez les Wauja, ces épisodes de maladie peuvent aussi initier une grande fête de masques (Barcelos Neto 2004). Murphy notait déjà l'extrême proximité formelle de la fête du manioc et du kwarup observé par Oberg (1953, p. 56) chez les Kamayura. Ne disposant encore que de données lacunaires, il indique : « si les cérémonies observées par Quain dans le village trumai et par Oberg chez les Kamayura sont différentes, elles témoignent d'une très grande similarité des formes rituelles pour des finalités religieuses différentes ${ }^{18}$ » (Murphy et Quain 1955, p. 67, note 5).

La tendance actuelle serait plutôt à une dissolution de la fête du manioc dans le script du rituel thérapeutique collectif, ce qui se traduit dans les narrations qu'on en fait par un raccourcissement de sa durée et une orientation davantage tournée vers la relation dyadique entre malade et hontal.

Ce contexte régional et historique une fois posé, nous allons nous intéresser maintenant plus précisément aux chants propres à ce rituel et à leur destinée au cours des dernières décennies. L'opposition notée plus haut, entre chants nocturnes et chants diurnes, se révèle centrale pour comprendre à la fois la disparition $\mathrm{du}$ rituel et la forme contemporaine de sa revitalisation.

\section{LES CHANTS NOCTURNES : UN « CORPUS ÉTENDU »}

Dans un village trumai où je passai quelques jours en janvier 2008, je fus témoin d'une énonciation surprenante, dans ce contexte, des chants du manioc. Elle émanait d'une très vieille femme, dont l'existence sociale était presque réduite à néant. Allongée dans son hamac dont elle repliait même de jour les pans sur son visage et son corps, elle passait ses journées depuis plusieurs mois à exhaler des chants rituels xinguaniens pour se « souvenir des moments de joie de son enfance et de son adolescence, et ainsi être gaie $"{ }^{19}$. Cette femme était la doyenne des Trumai, ne parlait pas un mot de portugais et était pour cela taxée de trumai ou d'adis (indien) t'axer, un morphème dénotant habituellement la colère ou la férocité, mais aussi, par extension, le débordement, l'excès par rapport au concept de référence. Elle était « ce qu'il y a de plus trumai » chez les Trumai d'aujourd'hui. Et elle chantait, entre autres, des chants du manioc (ole wal). Mais cette énonciation gratuite par une « pure » Trumai d'un chant survalorisé se faisait dans l'indifférence générale. Les raisons du déni de savoir qui frappait sa performance étaient partagées par tous les membres de la maisonnée. La grandmère «mentait » $(l a \tau)$. Ce terme désigne une erreur, pas forcément intention- 
nelle, mais dont l'auteur est néanmoins tenu pour responsable. Ce «mensonge » faisait sourire et discréditait l'ensemble du savoir rituel de la vieille dame. Plus précisément, son erreur consistait à chanter par bribes un savoir qui n'a de valeur qu'ordonné et complet. Les chants se succèdent dans un ordre prédéfini qui doit être scrupuleusement respecté. Or cette vieille femme les chantait manifestement dans le désordre, tels qu'ils lui revenaient en mémoire, et donnait même pour ole wal des chants d'autres rituels, comme le yamorikuma (rituel féminin). L'indifférence générale traduisait que sa performance n'était ni un rituel, ni même un enseignement possible : à quoi bon apprendre des chants épars lorsque leur valeur tient toute entière dans leur relation à une totalité close désormais perdue ? L'attitude de cette famille est représentative : ce répertoire ordonné a disparu sans appel avec la mort de l'ancien chef Amilcar, dans les années 1990.

Ce soin de l'ordonnancement séquentiel est caractéristique de certains grands rituels xinguaniens. Il signale l'existence d'un double niveau à prendre en compte pour caractériser ce savoir spécialisé : d'une part, un contenu - le chant proprement dit-, d'autre part, un ensemble de représentations additionnelles (un méta-savoir) sur les conditions dans lesquelles il doit être énoncé et appris. Il constitue donc ce que Déléage (2009) appelle un « savoir institué », l'institution étant définie par Sperber (1996, pp. 104-105) comme « un processus de distribution d'un ensemble de représentations, processus qui est gouverné par des représentations appartenant à cet ensemble même ". Cette définition est cependant trop générale pour caractériser l'institution de ces chants en particulier. Dans un article commun, Fausto, Franchetto et Montagnani (2011) établissent une distinction, utile ici, entre deux types de traditions ou "régimes de mémoire ». D’une part, le « corpus étendu », qui est « idéalement un ensemble fixe qui doit être répété à l'identique au fil du temps ». " Le changement y est vu comme une erreur du processus de transmission. Le régime de la mémoire n'est pas événementiel ou autobiographique, mais au contraire codifié et socialement distribué : il faut savoir qui se souvient de quoi, et comment » (ibid., p. 42). C'est le cas, par exemple, du Haut Xingu. D’autre part, dans certaines sociétés, « les individus se souviennent des situations qu'ils ont vécues, évoquent leurs expériences, mais ne sont pas formés à restituer une tradition. Ce qui s'apprend dans ces expériences fortement incorporées sont des schémas génératifs, extrêmement productifs, qui permettent de recréer indéfiniment le contenu de la tradition. C'est le cas par exemple des Parakanã, un peuple tupi guarani du Brésil, qui capturent continuellement, par le rêve, des chants toujours nouveaux qui ne seront jamais exécutés rituellement plus d'une fois » (ibid.).

Cette opposition est principalement, chez ces auteurs, l'outil d'une typologie entre sociétés. On en fera ici un usage différent, en remarquant qu'elle peut correspondre à une distinction épistémologique au sein d'une même société, voire d'un même rituel, quant au statut des chants, de leurs modes de transmission et de leurs contextes d'usage. Les critères de cette opposition ne sont pas les seuls 
possibles, mais il se trouve qu'ils correspondent remarquablement bien aux différences que les Trumai repèrent entre les deux genres vocaux du rituel du manioc. Les chants nocturnes relèvent nettement, d'après les Trumai d'aujourd'hui, du « corpus étendu » et s'apparentent ainsi, par leur épistémologie, aux chants du kwarup, du javari (hopep en trumai), du yamorikuma ou du rituel de flûtes masculin dont l'exécution est qualifiée de «chant» (wal) ${ }^{20}$. Chaque rituel associe aux chants à la fois un mythe et un script précis. Il forme donc un ensemble chorégraphique, musical et mythique (Basso 1985). Les chants eux-mêmes sont divisés en " suites ", pour conserver la terminologie en usage (Menezes Bastos 1990 ; Mello 2005 ; Fausto et al. 2011, p. 57 ; Piedade 2004), indexées sur un moment du jour ou de la nuit et correspondant à une unité chorégraphique. Ces suites se décomposent elles-mêmes en pièces, unités de mémorisation plus réduites. "Les suites sont ainsi indexées à des séquences d'actions rituelles, lesquelles ne sont pas rigidement alignées (tinapisi). À l'inverse les pièces qui composent une suite forment une série ordonnée unique et linéaire, dont la représentation traditionnelle est la suite de nœuds sur un cordon du palmier buriti, et, aujourd'hui, la liste numérotée dans les cahiers d'écolier des jeunes » (Fausto et al. 2011, p. 57).

Chez les Trumai, il est difficile aujourd'hui, pour le chant du manioc en particulier, de décider si le terme la (ou ela), le seul à désigner des unités musicales au sein des chants ( $w a l$ ), désigne les suites ou les pièces qui les composent ou, encore, les deux. Les autres sens du mot (chemin ou collier de perles en coquille d'escargot) penchent cependant vers la suite. La performance d'un de ces rituels repose en tout cas sur la mémorisation, par un spécialiste, d'un répertoire fini et considérable de chants. Cet apprentissage prend la forme d'un tête-à-tête entre professeur et apprenti, plusieurs heures par jour, et sur des périodes de plusieurs semaines ou mois s'étalant sur des années. Les spécialistes des chants (walke en trumai, littéralement « celui qui chante ») se transmettent ce savoir de père ou de grand-père à fils ou petit-fils, faisant ainsi émerger des lignées de chanteurs. Mais il est possible, en l'absence d'une telle relation de parenté, d'acquérir ce savoir moyennant un paiement élevé. Ces chants se caractérisent, pour la plupart des locuteurs, par leur opacité : ils sont souvent dans une langue étrangère (c'est le cas du javari pour les Trumai, apporté par eux, mais de langue tupi), qui a pu subir des modifications phonétiques dues à ces transmissions « à l'aveugle » et qui présente également des archaïsmes caractéristiques du langage rituel. La fidélité scrupuleuse à la tradition s'enracine, comme dans le javari, dans un mythe qui relate les modalités du premier apprentissage. L'ensemble de ces métareprésentations sur la distribution du savoir rituel, qui le circonscrit de fait à un petit nombre de spécialistes chargés de prestige, est au contraire extrêmement partagé. Tous savent dans quelles circonstances et selon quelles modalités l'on acquiert un répertoire rituel, tous savent l'importance de l'ordre. La traduction, lors de la performance, de cette valorisation par la rareté, le secret et le prix 
d'acquisition est le relief considérable accordé à l'erreur. Elle est toujours jugée marawan, c'est-à-dire présage de mort ou de maladie pour le chanteur ou l'un de ses proches. Elle relève de deux catégories : la confusion séquentielle et ce qu'on pourrait appeler la fausse note (au sens le plus large). La première est moins saillante que la seconde. Sauf inversion flagrante et repérable par tous, elle tient le plus souvent, dans les faits, à des lignes de transmission différentes et débouche sur des commentaires en forme de compétition d'autorité entre chanteurs et entre villages, chacun affirmant disposer du chant ou de l'ordre «correct». Rien de commun avec la stupeur fascinée que provoque une hésitation, un bégaiement, une langue qui fourche subitement ou, pire, le silence qui suit ces erreurs avant de reprendre. En Trumai, on emploie l'expression wal par (littéralement « chanter diviser »), tout comme le lapsus langagier est désigné par celle de ami par (« parler diviser »). Cette subite interruption du flux de parole, immédiatement décelable, y compris par le néophyte, provoque un malaise immédiat, chez le chanteur comme dans l'assistance, doublé d'une inquiétude sur son avenir proche. Les malheurs qui s'abattent sur lui sont, pour certains rituels, interprétés comme une vengeance de l'« esprit » (denetsak) rendu présent par la cérémonie et le chant.

Les chants nocturnes du manioc relèvent clairement aujourd'hui de cette catégorie. Ils sont opaques, dans une langue alternant, selon Murphy et Quain (1955), caribe et tupi. Ils sont de loin les plus prisés. Leur appellation est non marquée : c'est eux que l'on entend implicitement par l'expression ole wal. La situation actuelle de ces chants est celle d'un décalage mémoriel entre l'institution et l'institué : on ne se souvient plus des chants, mais tous savent qu'ils ont le statut de « corpus étendu ». Plus encore, la connaissance de cette institution accélère l'oubli : si beaucoup de vieux savent quelques strophes - en général les mêmes -, nul n'a le statut public d'héritier de la tradition. Tous savent que nul n'est autorisé à affirmer que son savoir constitue une totalité ordonnée. Les chants perdent dès lors toute valeur, leur énonciation tout sens, et une génération suffit à leur disparition complète.

\section{LES CHANTS DIURNES : COMPOSITION CONTEXTUELLE}

Le rituel du manioc était dans la journée le théâtre de chants très différents par leur épistémologie. Ces chants du jour (tamulan chïk) sont individuels et ouverts à l'invention. On dit en trumai qu'ils sont « faits avec la tête » (kutea letsi) pour décrire cette faculté d'imagination. Leur énonciation est distinguée du registre chanté (wal), puisqu'on la désigne par le verbe xom, traduit d'ordinaire par le portugais chupar (« sucer»). Il est employé pour la consommation de certains aliments (comme les fruits, le miel, les bonbons) et l'inhalation ou l'ingestion de tabac. Ce type de chants est aussi désigné par la locution wal ami («chanter parler»), qui explicite le fait que ces chants sont, à la différence des 
chants nocturnes et collectifs, faits pour être compris et qu'ils sont des messages adressés à des destinataires. La plupart des Trumai portent sur cette partie du rituel un jugement négatif. Ces chants parlés sont jugés «pas bons » (ae tak), c'est-à-dire à la fois socialement et moralement mauvais. On n'en rappelle pas le contenus exacts. Les restitutions qu'on en donne sont des reformulations simplifiées. Une vieille femme du poste Leonardo raconte par exemple :

Ils chantaient plein de choses. À leur ennemi, à celui qui est fâché, c'est à lui qu'ils " parlent-chantent », au sorcier, à celui qui veut les tuer. Il y en a qui fabrique une figurine d'humain avec de la cire, et ils dansent avec en disant : « avec cette abeille je crois que vous allez me tuer ». Tu peux aussi prendre un faisceau de flèches sur l'épaule et chanter : « avec ça vous allez me tuer, vous allez me flécher ». C'est vraiment pas bon ce qu'ils « parlent-chantent ». C'est à leurs ennemis qu'ils chantent.

L'abeille désigne par métaphore les sorts oke qui sont l'instrument principal de l'agression sorcellaire et que le chamane déterre et détruit sous l'œil de ses clients. Agressions guerrière ou sorcellaire sont donc les thèmes dont on se souvient principalement dans des restitutions qui sont des commentaires moraux sur le dicible et l'indicible aujourd'hui. Les chants diurnes sont donc davantage vus comme un rituel d'exacerbation des conflits que comme un dispositif de conciliation. Leur mise à distance rappelle l'amnésie complète qui frappe une autre institution proprement trumai, notée par Murphy et Quain (1955, p. 58), mais trop éloignée des valeurs de paix et de maîtrise xinguaniennes pour qu'on s'en souvienne aujourd'hui, celle des duels d'insultes sur la place du village.

Or, dans un village trumai, Cachoeira, dominé par la figure de sa doyenne Benedita, on adopte vis-à-vis de ces chants une attitude différente. Ils sont rappelés avec précision, non en vertu d'un apprentissage formalisé, mais parce qu'ils font appel à une mémoire sociale et événementielle. Il est ainsi possible de les caractériser plus finement. On commencera par les restituer avec leur signification et leur interprétation.

\footnotetext{
1 Pïx ha yo yi, tahay, ha-eta $\quad$ Ma verge est grosse, jeune fille, pauvre de moi Amale iyepika ha yets tsile Car je suis le fils d'Amale, à ce qu'on raconte
}

Chantés par un jeune homme qui voyait ses avances amoureuses repoussées par de nombreuses jeunes femmes, ces deux vers élégiaques sont une allusion au mythe d'origine du pequi, un fruit symbole de fertilité dont la récolte est marquée par un rituel important ${ }^{21}$. Amale est le nom du crocodile mythique dont le pénis, après sa mort, donna naissance à l'arbre.

\begin{tabular}{l|l|l}
2 & $\begin{array}{l}\text { Ha yo tsima tsima werew, di pa } \\
\text { Kurukuru mïxa kefane }\end{array}$ & Veuillez vous enfiler mon sexe, femmes \\
Telle la gorge du kurukuru
\end{tabular} 
Ce chant semblait s'être stabilisé. Inventé en une occasion précise dont Benedita n'a pas connaissance, il était mobilisable par tout homme qui voulait exprimer à une ou plusieurs femmes son dépit et sa frustration.

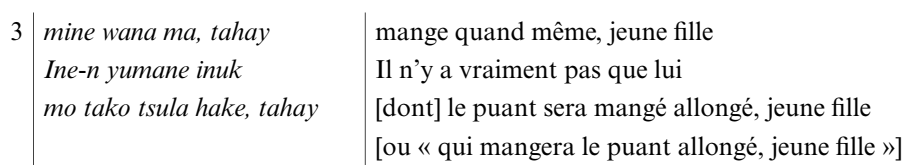

Cette strophe critiquait la naïveté d'un homme qui se croyait le seul amant d'une femme et, en même temps, cette femme pour son intempérance sexuelle. Il laisse ouvertes deux interprétations, chacune adressée plus particulièrement à l'un des protagonistes. Selon la première, l'énonciateur invite la jeune femme à l'échange sexuel en la priant de [le] manger "quand même » (par l'adverbe archaïque mine), sous-entendant par là qu'elle a déjà un amant, ce qui devrait la disposer au refus. Les deux vers suivants insistent sur l'appétit de la jeune fille, qui lui fait accepter jusqu'à une nourriture " puante », c'est-à-dire des partenaires peu attirants. La seconde interprétation, toujours fournie par les Trumai, porte sur les deux derniers vers seulement. Elle est permise par le fait que l'agent du verbe « manger » (tako) n'est pas exprimé. Il est donc possible d'inverser homme et femme pour imaginer que c'est cette dernière qui est désignée par le verbe substantivé $m o^{22}$. Et la moquerie s'adresse alors à l'homme qui accepte une nourriture consommée par d'autres avant lui, donc « puante».

\begin{tabular}{|c|c|}
\hline $\begin{array}{l}\text { tïkanïr ae pütï, deta } \\
\text { taf dulakes tam hen } \\
\text { helaka yi falxo tsula }^{23}\end{array}$ & $\begin{array}{l}\text { c'est lui en fait qui est fourmi géante, neveu } \\
\text { alors même que son cordon saigne encore } \\
\text { le village entier répand des rumeurs allongé }\end{array}$ \\
\hline
\end{tabular}

La fourmi tucandira est associée à la sorcellerie. L'initiation à cette spécialité passerait par des piqûres aux extrémités. Le rituel de contre-sorcellerie met en œuvre une étape d'ordalie où ces mêmes fourmis, cuites sur une plaque à cuire, sont les substituts des sorciers. Le premier vers exprime une accusation contre un tiers, prononcée par un oncle face à son neveu utérin. L'adverbe pï $\ddot{\imath}$ est un attributif qui implique un revirement par rapport à une croyance antérieure. Il implique donc que cette parole accusatrice en remplace une autre, et sera sans doute substituée elle-même par une troisième. Les deux vers suivants s'extraient de cette interlocution initiale pour traduire la pensée du chanteur, sous la forme d'une description ironique de la circulation des rumeurs de sorcellerie : le village « parle et accuse » (par le verbe archaïque falxo) jusqu'aux nouveaux-nés (dont le cordon ombilical n'est pas encore cicatrisé).

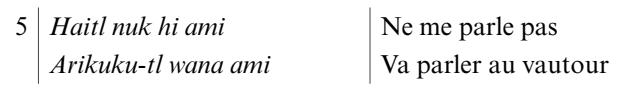




$$
\begin{array}{|l|l}
\text { Lofef-etl wana ami } & \text { Va parler à l'urubu noir } \\
\text { Deniktetl wana ami } & \text { Va parler à l'urubu roi }
\end{array}
$$

Ce chant était la réponse d'un assassin aux parents de sa victime qui lui adressaient des menaces et des reproches. Sûr de son bon droit, il invitait ses ennemis à parler aux oiseaux charognards qui étaient désormais les plus à même de les informer sur l'état de leur fils.

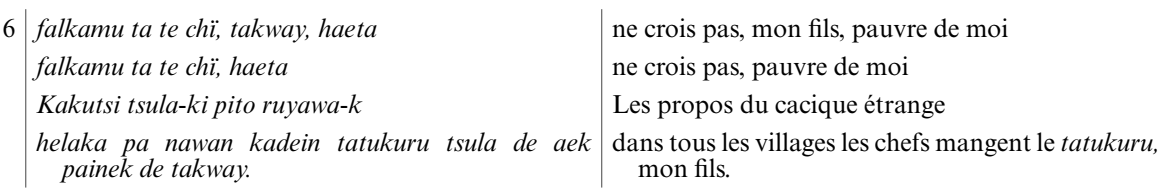

Ce chant était une réponse aux médisances et aux rumeurs. Benedita l'explique en ces termes :

Dans les autres villages on dit : « lui n'est vraiment pas bon, c'est lui qui est mauvais. Mais nous non, nous sommes bien ", c'est ce qu'ils disent. Il l'entend. Il entend ce qu'ils disent de lui. Alors il se venge d'eux. "Ne les crois pas mon enfant! Contente-toi d'écouter! Les chefs dans tous les villages aussi mangent du tatukuru».

Le cacique fait partie des oiseaux porteurs de nouvelles, plus souvent mauvaises que bonnes, et il figure ici la rumeur. Le tatukuru est une nourriture rituelle qui désigne métaphoriquement la faute dont est accusé à tort l'énonciateur.

Ces énoncés ont subi une double formalisation. Par la musique d'abord. Dans le corpus recueilli, les chants se coulent seulement dans deux mélodies pré-définies, qui ont chacune leur propre structure de répétition. Les vers restitués ici sous forme condensée, qui est celle de la mémorisation sémantique, sont répétés lors de l'énonciation selon une logique paralléliste complexe qui rappelle celle qui préside à la mise en souffle des incantations thérapeutiques. Ainsi le dernier chant, qui ne comporte que quatre vers sémantiques, est énoncé dans sa version chantée de la manière suivante :

Falkamu ta te chi

Falkamu ta te chï, takway

Falkamu ta te chï

Falkamu ta te chï

Kakutsi tsulaki pito ruyawak

He he he, he he he

Helaka pa nawan kadein tatukuru tsula de aek painek de takway

Helaka pa nawan kadein tatukuru tako tsula de, aek painek de takway

Falkamu ta te chï
Ne crois pas

Ne crois pas, mon fils

$\mathrm{Ne}$ crois pas

Ne crois pas

Les propos du cacique étrange

he he he, he he he

Dans tous les villages les chefs mangent le tatukuru, mon fils

Dans tous les villages les chefs mangent le tatukuru, mon fils

Ne crois pas 
Falkamu ta te chï takway, haeta

Falkamu ta te chï

Falkamu ta te chï takway, haeta

He he, he he,

Falkamu ta te chï,

Falkamu ta te chï, takway

Falkamu ta te chï

Falkamu ta te chï

Kakutsi tsulaki pito ruyawak

He he he, he he

Helaka pa nawan kadein tatukuru tako tsula de

Helaka pa nawan, takway

Helaka pa nawan kadein tatukuru tako tsula de

Helaka pa nawan, takway

Falkamu ta te chï,

Falkamu ta te chï, takway, haeta

Falkamu ta te chi

Falkamu ta te chï

Falkamu ta...

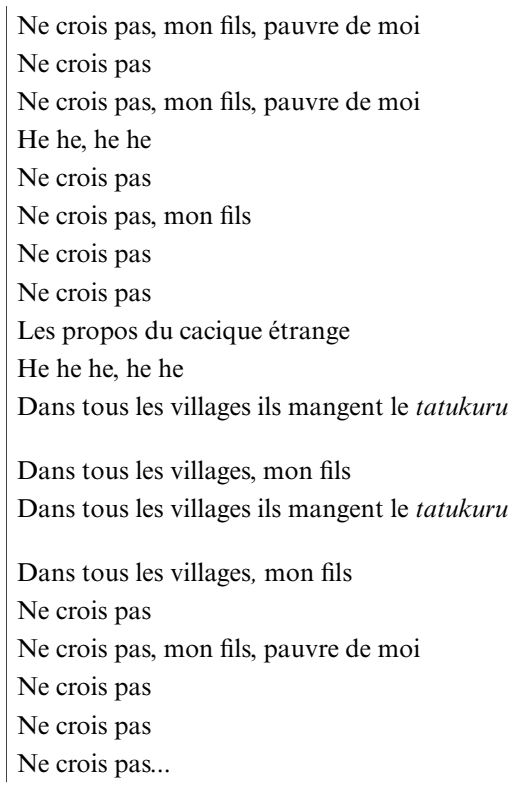

La langue elle-même est parfois différente du trumai ordinaire, selon plusieurs procédés :

- des " archaïsmes", soit des termes que les locuteurs actuels comprennent seulement par le contexte et par les explications fournies par Benedita, soit des usages rares de lexèmes courants (comme l'utilisation aspectuelle itérative du postural tsula, "allongé », auquel on préfère dans la langue courante le verbe laketsi, « se promener »).

- une simplification syntaxique (absence des marques de substantivisation, absence de marques casuelles ou locatives).

- des élisions de morphèmes ou de phonèmes, comme dans le premier vers du chant, que Benedita a complété, pour l'expliquer, par: Fal kamu ta(k) te(nuk hi) chï.

- des substitutions lexicales métonymiques et/ou métaphoriques (fourmi pour sorcier, manger pour copuler, etc.) qui sont canoniques, mais conservent parfois une polysémie.

Toutes ces spécificités servent une obligation manifeste de rendre le discours allusif et oblique, que l'on note même lorsque syntaxe et lexique sont respectés. C'est le cas du chant le plus simple, inventé par le frère de Benedita lors de la mort de leur père. Destinataire de rumeurs divergentes sur l'identité du sorcier responsable, il chanta dans chaque maison un unique vers : ha hup tak (« je ne sais pas »). Les destinataires du chant ne sont jamais spécifiés dans les formules impératives (« va parler au vautour! ») et, dans les assertions à la troisième personne, c'est un 
collectif anonyme, qui est mis en position de sujet ( le village entier répand des rumeurs »), ou un oiseau (le cacique). Ce refus de l'adresse directe se retrouve dans la chorégraphie rituelle : le chant est proféré dans toutes les maisons à tour de rôle, le point de départ et d'arrivée étant stipulé également.

Fait pour parler à ses ennemis, le rituel met en scène une parole qui s'adresse au contraire à tous. Il ne consiste donc pas en un passage mécanique d'un savoir partagé (où chacun sait) à un savoir public (où chacun sait que chacun sait), passage qui correspond à la différence entre une rumeur accusatrice et une accusation frontale devant témoins. Ce passage est en fait signalé sans jamais être franchi. Chacun entend un chant, et sait que tous l'entendent aussi, le cercle du village devenant, comme dans beaucoup de rituels, la projection spatiale d'une distribution publique du savoir (Chwe 1998). Mais l'identité du destinataire reste protégée par l'oblicité du discours et la chorégraphie rituelle. Voir dans ce caractère allusif une simple obéissance à un idéal moral est réducteur. En exigeant une compréhension du message qui est toute entière une interprétation pragmatique, donc en forçant les auditeurs à un regard réflexif sur le microcontexte social, le chant redouble son efficacité. Il critique et accuse sans nommer, neutralisant ainsi les potentialités conflictuelles immédiates, et crée de l'ironie et de la connivence par le rire sur le dos du destinataire.

Une des conséquences de ce caractère allusif est la polysémie de ces chants et, du coup, leur utilisation dans un grand nombre de situations concrètes. Autrement dit, si tous ont le statut de chants inventés et si l'on est toujours libre d'en proposer de nouveaux pour commenter tel conflit précis, certains font tradition car ils dépassent le contexte singulier qui a suscité leur composition. Il y aura toujours des hommes éconduits et des femmes jugées frivoles. La performance rituelle repose alors sur un choix dans un répertoire connu de tous, le choix étant alors en lui-même le message adressé à l'ennemi, au rival ou à l'amante. Certains chants semblent même en mesure de dépasser le domaine de conflit auquel Benedita les rattache de façon univoque. Ainsi le dernier chant, dont la formule finale: "tous les chefs mangent du tatukuru », est très indéfini. Si le registre alimentaire renvoie typiquement à la sexualité, le tatukuru désigne aussi une variété de tabac "féroce » (yuraw) dont la consommation est fatale et auquel on impute la mort de certains chamanes à la suite d'une tromperie. Un homme accusé de sorcellerie pourrait sans peine mobiliser cette implication pour renvoyer le soupçon à ses expéditeurs.

\section{UN NOUVEAU RITUEL POUR DE VIEUX CHANTS}

Le défaut de culture déploré par les Trumai s'est trouvé momentanément comblé en 2006 lors de l'inauguration d'une infirmerie dans le poste FUNAI de Pavuru. De façon intéressante, ce sont les chants diurnes qui ont été mobilisés 
pour l'occasion. Benedita les a répétés avec une fidélité scrupuleuse - de la même façon qu'elle les avait récités pour que je puisse les enregistrer quelques mois plus tôt -, assise, pendant une quinzaine de minutes, devant une assistance composée de responsables de la santé (médecins et infirmières de l'école de médecine de São Paulo) et d'une majorité d'Indiens ressortissant de ce poste, c'est-à-dire des Trumai, des Kamayura, des Kayabi, des Ikpeng et des Suyá. Les inaugurations de structures collectives sont devenues des rituels interethniques à part entière, pendant lesquels les groupes participants présentent à tour de rôle un échantillon spectaculaire de leur répertoire rituel. Les Trumai qui n'habitent pas avec Benedita ont critiqué sa prestation, qu'ils y aient assisté ou en aient entendu parler ensuite. Ils lui reprochaient de s'être trompée en confondant les deux types de chants, en donnant pour chants véritables (pi'tsi) ceux qui sont « inventés ». Benedita connaissait naturellement parfaitement cette dichotomie et savait à quelle classe appartenaient les chants qu'elle avait exécutés ce jour-là. L'erreur n'en serait pas moins présente aux yeux des autres, puisque Benedita modifie radicalement le contexte d'énonciation. Au lieu d'une pluralité de chanteurs choisissant chacun un chant référant à une situation singulière et parcourant le cercle du village, elle enchaîne tous les chants qu'elle connaît, sans bouger, au milieu d'une assistance qui n'en comprend pas le contenu sémantique. Benedita érige donc ces chants contextuels en patrimoine valorisé dans le rapport aux autres ethnies et aux Blancs. Ils semblent passer ici d'une épistémologie de l'invention et de l'ad hoc à une épistémologie du « corpus étendu ». Ce glissement se traduit par le souci, manifesté par Benedita, de se référer à une autorité. La notion d'erreur n'a normalement pas de sens pour les chants diurnes. Pourtant, dans les enregistrements que j'en ai faits, elle mentionne, comme dans les mythes, la source de son savoir : « je te raconte comme me l'a raconté ma mère ». Cette logique filiative se poursuit aux générations suivantes puisque, avant moi, l'un des fils de cette femme avait déjà enregistré ces mêmes chants, ainsi que la linguiste Raquel Guirardello Damian dans les années 1990. À chaque fois, Benedita donna les mêmes explications et invoqua l'autorité de sa mère pour accréditer son savoir.

Comment expliquer ce glissement d'une épistémologie à une autre ? Le village de Cachoeira est celui des Awaldat, ethnonyme d'un sous-groupe qui aurait rejoint le village trumai peu avant l'arrivée de Quain, pour faire face à la dépopulation massive liée aux épidémies et aux attaques ennemies. Si, par la généalogie, plus aucun Trumai n'est un « pur » Awaldat, le terme continue d'être attribué de manière péjorative à ceux de leurs descendants qui appartiennent à une faction politique minoritaire, dont on suit la perpétuation depuis Quain. Les Awaldat étaient cependant Trumai par la langue, même si les autres continuent d'invoquer de micro-variations idiomatiques et de prononciation anciennes pour affirmer leur étrangeté. Aujourd'hui, les habitants de Cachoeira revendiquent l'ethnonyme Awaldat, au point d'avoir nommé ainsi leur école. 
Après qu'Amilcar eut succédé au père de Benedita, il y a une cinquantaine d'années, cette faction a été affaiblie. Lui et ses descendants directs ont privilégié, par les choix matrimoniaux comme par les choix de résidence, le rapprochement avec le Bas Xingu, notamment les Suyá et les Kayabi ${ }^{26}$. Les autres Trumai, à la suite d'Amilcar, lui-même fils d'une femme kamayura, se sont rapprochés du Haut Xingu. Les Awaldat ont cessé de vivre avec eux, d'abord en restant dans les villages de leurs alliés ou dans le poste Diawarum, qui se trouve dans le Bas Xingu, puis dans leur propre village à partir de 1984. Depuis lors, la micro-unité villageoise constituée d'une paire de germains (Benedita et son frère) et de leurs enfants a progressivement étoffé ses rangs et conquis une forme d'aisance relative. Ce village est aussi plus engagé que les autres dans une certaine forme de modernité indigène, qui passe par l'école et les ONG. Ceci s'explique en partie par l'alliance de Benedita avec un homme kayabi qui était fonctionnaire de la FUNAI. L'argent versé à sa veuve, après son décès, a permis de faire construire la maison en briques et tuiles qu'elle habite aujourd'hui et qui est une exception dans le parc, ce genre de construction étant réservé aux infrastructures collectives dans les villages, école ou infirmerie, et aux bâtiments des postes de la FUNAI. Le village est désormais bem organizadinho («bien organisé »), avec sa citerne et sa pompe alimentée par des panneaux solaires, sa radio en état de marche, son groupe électrogène et sa parabole. Les Kayabi, arrivés dans le Parc après une longue histoire de relations avec les Blancs, sont aujourd'hui responsables du succès de l'association ATIX (Associação Terra Indigena Xingu), qui mène divers programmes de surveillance des frontières et de développement local (par l'artisanat et le miel essentiellement), en collaboration avec l'ISA (Instituto Socio Ambiental, la plus importante ONG indigéniste brésilienne). Le second fils de Benedita y occupe un poste important. Et sa maison confortable dans la ville de Canarana sert de tête de pont au village. L'ISA conduit aussi, depuis une quinzaine d'années, un projet de scolarisation, en formant des professeurs indigènes et en publiant du matériel pédagogique en langue indigène. Ce projet est un opérateur puissant de la patrimonialisation culturelle et linguistique, et c'est à Cachoeira, village où le trumai est le moins parlé, qu'il a eu le plus d'impact. José, fils cadet de Benedita, a suivi les formations de l'ISA depuis le début et est devenu un lettré autochtone, figure désormais nationale dont Kohler (2009) a donné récemment une description très juste. Il a appris la langue trumai qu'il ne connaissait pas (il dit qu'avant cela il était un Kayabi et que, désormais, il se sent Trumai) et il l'enseigne aux enfants et aux adolescents dans le cadre scolaire. Sa maison regorge de carnets annotés et de livres pédagogiques, sa télévision croule sous les DVD et les cassettes de rituels xinguaniens.

Ce détour permet d'éclairer le glissement épistémologique subi par les chants diurnes. En premier lieu, ce n'est pas un hasard si ce sont les Awaldat qui l'ont opéré, étant plus avancés que les autres sur le chemin de la patrimonialisation. En second lieu, la fracture politique qui les sépare des autres Trumai est suffisante 
pour qu'ils ne subissent pas au même degré l'autorité posthume d'Amilcar et acceptent d'affronter les désaccords de ses descendants. Ils n'ont pas été jusqu'à ressusciter les quelques chants nocturnes dont on se souvient encore et dont Amilcar est perçu, même par les Awaldat, comme le dernier connaisseur. Mais les chants diurnes étaient disponibles pour ce coup de force car ils n'avaient pas le même statut, étant jugés sans valeur et sans détenteur.

\section{Conclusion}

Cet épisode ponctuel de « revitalisation » prend ainsi place dans un double mouvement d'agrégation interethnique (avec le Bas Xingu) et de scission (d'avec les autres Trumai). Ce soubassement sociologique le fait accéder à un niveau supplémentaire de complexité, puisqu'il a en définitive deux types de destinataires.

Avec le Bas Xingu, il s'agit clairement d'un phénomène d'invention de la culture. Les habitants de Cachoeira démontrent à leurs partenaires désormais principaux (par l'alliance et les projetos), qui ne parlent pas le trumai, la possession d'un savoir rituel « en propre », critère inévitable d'indianité. Carneiro da Cunha (2010) a proposé un approfondissement récent de ce type de phénomène, en opposant culture (sans guillemets) et «culture». Si, la première est définie comme une « logique intérieure à chaque culture », la seconde est « une logique interethnique, pour laquelle chaque culture n'est qu'un des éléments du système » (ibid., p. 74). Comme elle le précise, « cela ne veut pas dire que leur contenu est nécessairement différent. Je pense plutôt qu'elles appartiennent à deux univers de discours différents, ce qui n'est pas sans conséquence » (ibid., p. 9). Elle remarque à juste titre que ces processus ne sont pas nécessairement propres à des relations politiques déséquilibrées, mais procèdent d'une structure logique plus fondamentale. Ce retour à ce dénominateur commun qu'est l'interethnicité permet de se dégager partiellement de la seule problématique de la patrimonialisation pour observer ce qui, dans un événement comme celui qui nous a intéressés, relève d'une tendance plus ancienne. Si les outils employés (vidéos, cassettes audio, écriture) pour fixer ce savoir sont indéniablement des emprunts et qu'ils permettent à l'échelle mondiale une forme de congélation patrimoniale des cultures autochtones, rien ne dit que les fins poursuivies soient similaires à celles qui règnent à l'UNESCO. La forme de muséographie autodidacte à laquelle se livre José est-elle si différente de l'entêtement qui a poussé certains individus exceptionnels, à chaque génération, à apprendre un corpus de chants immense? Est-elle même seulement une muséographie ? Les intenses répétitions rituelles des deux dernières années montrent combien il est devenu, par ce travail scripturaire, un des artisans d'une récupération effective qui permet d'inviter les autres villages, en particulier trumai. Il faudrait, tout en rejetant pour trop modernes les 
changements actuels, pouvoir expliquer les mécanismes qui ont conduit à la constitution de ces immenses répertoires de chants xinguaniens par le passé. Si la réponse à cette question dépasse largement notre cadre, elle passe sans doute par la prise en compte de contraintes socio-politiques comparables à celles qui font que des ragots moralement mauvais, vieux de 80 ans, puissent devenir un emblème sonore jugé « beau ».

Cette énonciation avait aussi pour destinataires les Trumai, seuls dans l'assistance à pouvoir comprendre les chants et mesurer les changements apportés au cadre rituel. Les commentaires que me firent les habitants de Cachoeira sur la performance de leur leader témoignent qu'ils en étaient d'ailleurs parfaitement conscients. S'ils insistaient en premier lieu sur la dimension esthétique (révélant ainsi le registre désormais principal d'appréciation, avant celui de la signification), ils prenaient tous systématiquement les devants sur les reproches qu'ils savaient devoir subir en sous-main de leurs parents trumai. L'un d'entre eux m'expliqua par exemple :

Je sais que les gens critiquent, qu'il y en a qui disent que ce n'est pas comme ça ou comme ci. Mais on les laisse parler. Nous, on fait, on n'est pas perdus, on sait où on va. Il est facile de critiquer quand on ne fait rien.

La logique de communication à l'œuvre ici n'est donc finalement pas si différente de celle qui présidait aux énonciations originelles des chants diurnes. De manière oblique et implicite, en faisant référence à des rumeurs et des tensions politiques internes, le fait même de cette performance innovante est un message allusif adressé aux Trumai sur ce que sont la tradition et la cultura. La véritable autorité de la tradition, c'est précisément peut-être de s'en emparer autoritairement, et non de s'en réclamer seulement par un respect scrupuleux à une ligne de filiation. Sortir du stigmate de la société sans culture est à ce prix. *

* Manuscrit reçu en novembre 2009, accepté pour publication en janvier 2011.

\section{NoTeS}

Cet article a été écrit grâce à une bourse du musée du quai Branly. Merci à Pierre Déléage, François Berthomé et Aurore Monod Becquelin pour leurs conseils et leurs relectures, ainsi qu'à Raquel Guirardello et Bruna Franchetto pour avoir rendu accessibles les photographies de Buell Quain.

1. Les noms propres, anthroponymes comme toponymes, ont été modifiés pour préserver l'anonymat des personnes.

2. FUndação NAcional do Índio (Fondation nationale de l'Indien).

3. Le décompte des Trumai est évidemment problématique compte tenu de l'entrelacement matrimonial qui fait de l'appartenance ethnique une affaire hautement contextuelle. En additionnant les habitants des différentes poches résidentielles (villages, postes frontières, familles parlant trumai dans un poste FUNAI ou en ville), on peut estimer entre 150 et 200 individus la population trumai actuelle.

4. Les caractères trumai se lisent comme en français sauf les suivants :' : consonne occlusive glottale; $\tau$ : consonne occlusive alvéolaire (mais avec une pointe de rétroflexe) ; $\mathrm{x}:$ consonne fricative 
vélaire (comme le $j$ espagnol) ; r : consonne battue alvéolaire (comme le $r$ de caramba en portugais) ; $\mathrm{h}$ : consonne fricative glottale (comme dans house); e : voyelle moyenne antérieure (comme dans éthéré) ; i : voyelle fermée centrale ; $\mathrm{u}$ : voyelle postérieure fermée (comme dans chou) ; w : semivoyelle labiale (comme dans wok).

5. Sur la notion de "cultura » et la patrimonialisation chez les Trumai (Monod Becquelin et al. 2008 ; Vienne et Allard 2006).

6. Un des jingles de la chaîne Globo, en 2005, était un court dessin animé représentant un indien du Haut Xingu sortant d'une sorte de hutte pour sautiller en poussant des cris autour d'un semblant de poteau de kwarup.

7. "Its purpose was essentially to insure an abundant manioc crop, and much of the symbolism involved was clearly oriented around the concept of fertility ». Toutes les traductions en français ont été faites par mes soins, le texte original est donné en note.

8. Les Trumai prononcent nukekerihe.

9. Crédits pour toutes les photos de cet article : Setor de Etnologia do Departmento de Antropologia, Museu Nacional, Universidade Federal do Rio de Janeiro.

10. " [...] but in the present context it applies to a temporary ceremonial identification between people, and between people and the ole posts. The interpersonal ties set up were only between people of opposite sex. However, a man could have more than one woman for aton, and vice versa ".

11. Le verbe employé pour décrire ce processus de " maîtrise ", au sens très précis de " donner un maître », procède en adjoignant au terme yar (maître) le suffixe causatif $-k a$. Le tronc, marqué à l'absolutif, est littéralement « fait maître » (c'est-à-dire qu'il en « reçoit un ») par un humain marqué au cas ergatif, qui dénote l'agent : yi yar-ka-n yaw-ak (particule maître-causatif-3Abs humain-ergatif).

12. Se reporter, par exemple, aux travaux de Barcelos Neto (2004) et de Piedade (2004) chez les Wauja.

13. Même si Murphy et Quain (1955) ne l'indiquent pas, il est assez probable que le roucou utilisé est la variété féminine murucuyu et non le madod masculin.

14. "On one occasion Matiwana performed a ritual in which he was apparently acting the part of Nukekerihe. He made wrathful noises and jumped about in front of the post, before which were two bowls of manioc soup. He then started a song about the post in which he was joined by Nituary and the women. After two repetitions, the women poured a bowl of the soup on Matiwana's head and shoulders, making a dripping mess of him. He retired amid general laughter. The second bowl of soup was then poured over the pole. A full explanation of the beliefs surrounding the ole posts was not obtained 》.

15. On ne sait pas si cette soupe est du maisawarï (Pt. perereba), boisson chaude et sucrée obtenue par ébullition du jus de manioc râpé, ou du wïlïx, soupe froide obtenue par désagrégation d'une galette de manioc.

16. " Fear of Nukekerihe was mixed with jokes".

17. "Quain was told one night that the men would not go to bed until he did, for if he stayed up long Nukekerehe would surely eat him ».

18. "If the ceremonies observed by Quain in the Trumai village and by Oberg among the Kamayura are not the same, a great similarity of ritual forms for different religious purposes is indicated ».

19. La forme singulière de cette pré-agonie (elle est décédée un an et demi plus tard) condense de manière saisissante plusieurs éléments clés de l'ethnologie régionale. L'importance du registre chanté dans la mémoire individuelle d'abord : les grandes fêtes (deani) interethniques et, plus précisément, les chants qui en constituent le cœur sont visiblement parmi les moments les plus mémorables d'une existence et les plus denses en joie (la finalité explicite des rituels en général est d'égayer). Cette énonciation non rituelle d'un chant rituel illustre aussi la nature essentiellement sociale et relationnelle, en Amazonie, de la maladie et de la mort (Taylor 1996). Socialement presque déjà défunte, elle maintenait à distance par cette évocation festive le ressassement mélancolique (faxla) caractéristique de l'étiolement des relations avec les consanguins et facteur important de la maladie, puisque cet état attire les esprits pathogènes denetsak. D'un point de vue externe, cette évocation signale paradoxalement l'agonie, puisque les parents convoqués sont des morts. 
20. On se référera, entre autres, à Menezes Bastos (1990, 2003) pour le javari chez les Kamayura, Piedade (2004) pour les flûtes kawoka chez les Wauja, Mello (2005) pour les chants féminins iamurikuma dans ce même groupe, enfin à Franchetto et Montagnani (2009) pour le complexe kagutultolo.

21. C'est aussi une graine de pequi que les jeunes filles déposent au pied des chefs lors de la sortie de réclusion, lorsqu'elles sont à l'apogée de leur féminité.

22. Un verbe qui devrait normalement recevoir une marque de substantivisation. Cette lacune tient à la formalisation du discours propre au genre, voir infra dans le texte.

23. Le postural tsula a un sens aspectuel duratif.

24. Coragyps atratus.

25. Sarcoramphus papa.

26. Les Villas Bóas ont été ici des agents matrimoniaux importants. Ce fait n'ôte pas à cette inflexion vers le Bas Xingu tout caractère de choix. La preuve en est que les Awaldat ont été les seuls, ou presque, à être concernés par ces mariages « arrangés ». Autrement dit, il semble que les Awaldat aient profité de la politique pacificatrice des Villas Bóas pour consommer la scission factionnelle.

\section{RÉFÉRENCES CITÉES}

\section{Agostinho da Silva Pedro}

1974 Kwarup : mito e ritual no Alto Xingu, Editora da USP, São Paulo.

BALL Christopher

2011 "As spirits speak. Interaction in Wauja exoteric ritual», Journal de la Société des Américanistes, 97 (1), pp. 87-117.

BarCelos Neto Aristóteles

2004 Apapaatai : rituais de mácaras no Alto Xingu, thèse de doctorat en antropologie sociale, Universidade de São Paulo (USP), São Paulo.

BAsso Ellen B.

1985 A musical view of the universe: Kalapalo myth and ritual performances, University of Pennsylvania Press, Philadelphie.

CARneiro da Cunha Manuela

2010 Savoir traditionnel, droits intellectuels et dialectique de la culture, Éditions de l'Éclat, Paris.

\section{CHwe Michael Suk-Young}

1998 "Culture, circles, and commercials : publicity, common knowledge, and social coordination ", Rationality and Society, 10 (1), pp. 47-75.

\section{DÉLÉAGE Pierre}

2005 Le chamanisme sharanahua. Enquête sur l'apprentissage et l'épistémologie d'un rituel, thèse de doctorat en anthropologie sociale, École des hautes études en sciences sociales, Paris.

2009 Le chant de l'anaconda. L'apprentissage du chamanisme chez les Sharanahua (Amazonie occidentale), Société d'ethnologie, Nanterre.

Fausto Carlos

2004 O tempo do ritual : política, economia e xamanismo no Alto Xingu [projet de recherche], Museu Nacional/Universidade Federal do Rio de Janeiro, Rio de Janeiro. 
Fausto Carlos, Bruna Franchetto et Tommaso Montagnani

2011 «Les formes de la mémoire. Art verbal et musique chez les Kuikuro du Haut Xingu », L'Homme, 197, pp. 41-70.

FIORINI Marcelo et Christopher BALL

2006 «Le commerce de la culture, la médecine rituelle et le Coca-Cola », Gradhiva, 4, pp. 97-113.

Franchetto Bruna et Tommaso Montagnani

2009 " Musique et langage chez les Kuikuro du Haut Xingu », présentation au séminaire de Carlo Severi, musée du quai Branly, Paris [manuscrit].

KOHLER Florent

2009 «Du caboclo à l'indigène : réflexions sur l'ethnogenèse au Brésil », Journal de la Société des Américanistes, 95 (1), pp. 41-72.

Mello Maria Ignez Cruz

2005 Iamurikuma : música, mito e ritual entre os Wauja do Alto Xingu, thèse de doctorat en anthropologie sociale, Universidade Federal de Santa Catarina, Florianópolis.

Menezes Bastos Rafael José de

1990 A festa da jaguatirica : uma partitura crítico-interpretativa, thèse de doctorat en anthropologie sociale, Universidade de São Paulo (USP), São Paulo.

2003 «A festa da Jaguaratirica : primeiro e sétimo cânticos. Introdução, transcrição e comentários ", Ilha. Revista de Antropologia, 4 (2), pp. 133-174.

Monod BeCQuelin Aurore

1975 La pratique linguistique des indiens Trumai (Haut-Xingu, Mato Grosso, Brésil). Tome 1, Société d'études linguistiques et anthropologiques de France, Paris.

Monod Becquelin Aurore, Emmanuel de Vienne et Raquel Guirardello-Damian

2008 "Working together : the interface between researchers and the native people. The Trumai case ", in David K. Harrison, David S. Rood et Arienne Dwyer (éd.), Lessons from documented endangered languages, typological studies in language, John Benjamins publishing company, Amsterdam/Philadephia, pp. 43-66.

MurPHY Robert et Buell QUAIN

1955 The Trumai Indians of Central Brazil, J. J. Augustin Publisher, New York.

OBERG Kalervo

1953 Indian tribes of Northern Mato Grosso, Brazil, Smithsonian Institution, Institute of Social Antrhopology, Publication n ${ }^{\circ}$ 15, Washington.

PIEDAde Acacio Tadeu de Camargo

$2004 O$ canto do Kawoká : música, cosmologia e filosofia entre os Wauja do Alto $X i n g u$, thèse de doctorat, Universidade Fedral de Santa Catarina, Florianópolis.

SEEGER Anthony

1979 «What can we learn when they sing ? Vocal genres of the Suyá Indians of Central Brazil », Ethnomusicology, 23 (3), pp. 373-394. 
1987 Why Suyá sing? A musical anthropology of an Amazonian people, Cambridge University Press, Cambridge.

SPERBER Dan

1996 La contagion des idées, Odile Jacob, Paris.

STEINEN Karl von den

1886 Durch Central-Brasilien : Expedition zur Erforschung des Schingú im Jahre 1884, Brockhaus, Leipzig.

TAYLOR Anne Christine

1996 "The soul's body and its states : an Amazonian perspective on the nature of being human », The Journal of the Royal Anthropological Institute, 2 (2), pp. 201-215.

VIENNE Emmanuel de et Olivier AlLARD

2006 «Pour une poignée de dollars? Transmission et patrimonialisation de la culture chez les Trumai du Brésil central », Cahiers des Amériques latines, 48-49, pp. 127-145.

Villas Bóas Orlando et Cláudio Villas Bóas

1974 Xingu : the Indians, their myths, Souvenir Press Ltd, Londres. 